\title{
Parameter-free calculation of charge-changing cross sections at high energy
}

\author{
Y. Suzuki, ${ }^{1,2}$ W. Horiuchi, ${ }^{3}$ S. Terashima,${ }^{4}$ R. Kanungo, ${ }^{5}$ F. Ameil,${ }^{6}$ J. Atkinson, ${ }^{7}$ Y. Ayyad, ${ }^{7}$ D. Cortina-Gil,${ }^{8}$ I. Dillmann, ${ }^{6}$ \\ A. Estradé, ${ }^{5,6}$ A. Evdokimov, ${ }^{6}$ F. Farinon, ${ }^{6}$ H. Geissel,,${ }^{6}$ G. Guastalla, ${ }^{6}$ R. Janik, ${ }^{9}$ R. Knoebel, ${ }^{6}$ J. Kurcewicz, ${ }^{6}$ Yu. A. Litvinov, ${ }^{6}$ \\ M. Marta, ${ }^{6}$ M. Mostazo, ${ }^{8}$ I. Mukha,${ }^{6}$ C. Nociforo, ${ }^{6}$ H. J. Ong, ${ }^{7}$ S. Pietri, ${ }^{6}$ A. Prochazka, ${ }^{6}$ C. Scheidenberger, ${ }^{6}$ B. Sitar, ${ }^{9}$ \\ P. Strmen, ${ }^{9}$ M. Takechi,,${ }^{6,}$ J. Tanaka, ${ }^{7}$ I. Tanihata, ${ }^{4,7}$ J. Vargas ${ }^{8}{ }^{8}$ H. Weick, ${ }^{6}$ and J. S. Winfield ${ }^{6}$ \\ ${ }^{1}$ Department of Physics, Niigata University, Niigata 950-2181, Japan \\ ${ }^{2}$ RIKEN Nishina Center, Wako 351-0198, Japan \\ ${ }^{3}$ Department of Physics, Hokkaido University, Sapporo 060-0810, Japan \\ ${ }^{4}$ School of Physics and Nuclear Energy Engineering and IRCNPC, Beihang University, Beijing 100191, China \\ ${ }^{5}$ Saint Mary's University, Halifax, Nova Scotia B3H 3C3, Canada \\ ${ }^{6}$ GSI Helmholtz Center, 64291 Darmstadt, Germany \\ ${ }^{7}$ RCNP, Osaka University, Ibaraki 567-0047, Japan \\ ${ }^{8}$ Universidad de Santiago de Compostela, Santiago de Compostela, Spain \\ ${ }^{9}$ Comenius University, Bratislava, Slovakia \\ (Received 9 May 2016; published 5 July 2016)
}

\begin{abstract}
Charge-changing cross sections at high energies are expected to provide useful information on nuclear charge radii. No reliable theory to calculate the cross section has yet been available. We develop a formula using Glauber and eikonal approximations and test its validity with recent new data on carbon isotopes measured at around $900 \mathrm{~A} \mathrm{MeV}$. We first confirm that our theory reproduces the cross sections of ${ }^{12,13,14} \mathrm{C}+{ }^{12} \mathrm{C}$ consistently with the known charge radii. Next we show that the cross sections of ${ }^{12-19} \mathrm{C}$ on a proton target are all well reproduced provided the role of neutrons is accounted for. We also discuss the energy dependence of the charge-changing cross sections.
\end{abstract}

DOI: 10.1103/PhysRevC.94.011602

A study of unstable nuclei is one of the fields that have been promoted most intensively. Charge distribution or charge radius, among others, is one of the fundamental quantities to characterize the ground-state properties of nuclei. Electron scattering measurement is ideal for probing the distribution but so far not applicable to short-lived unstable nuclei. We note, however, that the electron-ion scattering experiment will be available in the near future, as planned in Refs. [1,2]. Isotope shift measurement allows us to precisely deduce the charge (proton) radius for some limited unstable nuclei. The measurement of the charge-changing cross section (CCCS) newly appears as a potential means to extract the proton radius since it has the great advantage that the cross section can be measured for almost all nuclei by the same setup as the total reaction or interaction cross section that plays a decisive role in determining the nuclear matter radius [3]. In fact the CCCS has recently been measured to get information on the proton radii of light unstable nuclei [4-8].

A theoretical tool for extracting the matter radius from the high-energy total reaction cross sections is well established with the help of Glauber theory [9]. See Refs. [10,11] for a useful application to determining both proton and neutron radii. The reaction mechanism for the charge-changing reaction (CCR) is, however, not well understood and energydependent adjustments are introduced to analyze the CCCS data [4-6,12], which makes it difficult to obtain proton radii from the measurement. The purpose of this paper is to show

\footnotetext{
*Present address: Department of Physics, Niigata University, Niigata 950-2181, Japan.
}

that recent new CCCS data of carbon isotopes on both ${ }^{12} \mathrm{C}$ [13] and proton targets are all satisfactorily reproduced in the framework of the Glauber and eikonal models. The role of neutrons becomes evident for the proton target. This is an important step toward constructing a method of analyzing CCCSs with the use of no adjustable parameters.

The total reaction cross section can be calculated by

$$
\sigma_{R}=\int d \boldsymbol{b}\left(1-\left|\left\langle 0\left|e^{i\left(\chi^{p}+\chi^{n}\right)}\right| 0\right\rangle\right|^{2}\right),
$$

where $\boldsymbol{b}$ is a two-dimensional (2D) impact parameter vector perpendicular to the beam $(z)$ direction, $|0\rangle=\left|0_{P} 0_{T}\right\rangle$ is a product of the projectile and target ground-state wave functions, and, e.g., $\chi^{p}$ is a sum of the phase-shift functions $\chi_{p N}$ between the projectile's proton $(p)$ and the target's nucleon $(N)$ :

$$
\chi^{p}=\sum_{i \in p} \sum_{j \in N} \chi_{p N}\left(\boldsymbol{b}+\boldsymbol{s}_{i}-\boldsymbol{t}_{j}\right) .
$$

Here $s_{i}\left(t_{j}\right)$ is the 2D coordinate, perpendicular to the $z$ direction, of the $i$ th ( $j$ th) nucleon of the projectile (target) from its center of mass.

We first derive a formula to calculate the CCCS $\left(\sigma_{\mathrm{cc}}\right)$ following the derivation of Eq. (1) $[14,15]$. After the projectiletarget collision at $\boldsymbol{b}$, the initial state changes to $e^{i\left(\chi^{p}+\chi^{n}\right)}|0\rangle$. We assume that the state of the projectile nucleus can be represented by a product of the proton and neutron parts, $\left|\Phi_{P}^{(p, a)} \Phi_{P}^{\left(n, a^{\prime}\right)}\right\rangle$, where $a$ and $a^{\prime}$ specify the proton and neutron states, respectively. The projectile ground state is $\left|0_{P}\right\rangle=$ $\left|\Phi_{P}^{(p, 0)} \Phi_{P}^{(n, 0)}\right\rangle$. Any states with $a \neq 0$ are assumed to emit at 
least one proton, leading to the CCR. The probability of the CCR reads

$$
P_{\mathrm{cc}}(\boldsymbol{b})=1-\sum_{a^{\prime}, \beta}\left|\left\langle\Phi_{P}^{(p, 0)} \Phi_{P}^{\left(n, a^{\prime}\right)} \Psi_{T}^{(\beta)}\left|e^{i\left(\chi^{n}+\chi^{p}\right)}\right| 0\right\rangle\right|^{2},
$$

where $\beta$ specifies the state of the target nucleus. With the use of the closure relation

$$
\sum_{a^{\prime}, \beta}\left|\Phi_{P}^{\left(n, a^{\prime}\right)} \Psi_{T}^{(\beta)}\right\rangle\left\langle\Phi_{P}^{\left(n, a^{\prime}\right)} \Psi_{T}^{(\beta)}\right|=1
$$

and the unitarity condition of the phase-shift function

$$
\left(e^{i \chi_{n N}}\right)^{\dagger} e^{i \chi_{n N}}=1,
$$

we obtain $P_{\mathrm{cc}}(\boldsymbol{b})$ as follows:

$$
P_{\mathrm{cc}}(\boldsymbol{b})=1-\left\langle 0_{T}|F(\boldsymbol{b}, \boldsymbol{\xi})| 0_{T}\right\rangle,
$$

with the projectile-proton "transparency function"

$$
F(\boldsymbol{b}, \boldsymbol{\xi})=\left|\left\langle\Phi_{P}^{(p, 0)}\left|e^{i \chi^{p}}\right| \Phi_{P}^{(p, 0)}\right\rangle\right|^{2},
$$

where $\xi$ stands for a set of all the coordinates of the target nucleons. Note that the $\chi^{n}$ is present in Eq. (3) but finally disappears in Eq. (6). Namely, only the interaction of the projectile's protons with the target nucleons leads to the CCR but the projectile's neutrons play no role. We call this process "direct" in what follows. The $\sigma_{\mathrm{cc}}$ corresponding to the direct process is given by

$$
\sigma_{\mathrm{cc}}^{\mathrm{dir}}=\int d \boldsymbol{b} P_{\mathrm{cc}}^{\mathrm{dir}}(\boldsymbol{b}) .
$$

The matrix elements in Eqs. (1), (6), and (7) are usually evaluated in the optical-limit approximation (OLA) $[9,15,16]$ that requires only the nuclear one-body densities. Within the OLA, we obtain

$$
\begin{aligned}
P_{\mathrm{cc}}^{\operatorname{dir}}(\boldsymbol{b})= & 1-\exp \left(-2 \sum_{N=p, n} \iint d \boldsymbol{s} d \boldsymbol{t} T_{P}^{(p)}(\boldsymbol{s}) T_{T}^{(N)}(\boldsymbol{t})\right. \\
& \left.\times \operatorname{Re} \Gamma_{p N}(\boldsymbol{b}+\boldsymbol{s}-\boldsymbol{t})\right),
\end{aligned}
$$

where, e.g., $T_{P}^{(p)}(s)$ is the thickness function of the projectile's proton density $\rho_{P}^{(p)}(\boldsymbol{r}), T_{P}^{(p)}(\boldsymbol{s})=\int_{-\infty}^{\infty} d z \rho_{P}^{(p)}(\boldsymbol{r})$ with $\boldsymbol{r}=$ $(\boldsymbol{s}, z)$. The $N N$ profile function, $\Gamma_{N N}=1-e^{i \chi_{N N}}$, is usually expressed using the $N N$ collision data at the incident energy $E$ as [17]

$$
\Gamma_{N N}(\boldsymbol{b})=\frac{1-i \alpha_{N N}}{4 \pi \beta_{N N}} \sigma_{N N}^{\mathrm{tot}} \exp \left(-\frac{\boldsymbol{b}^{2}}{2 \beta_{N N}}\right) .
$$

The values of $\alpha_{N N}, \beta_{N N}$, and $\sigma_{N N}^{\text {tot }}$ are given in Ref. [18] for a wide range of $E$. The zero-range approximation simplifies $\Gamma_{N N}(\boldsymbol{b})$ to $\frac{1}{2}\left(1-i \alpha_{N N}\right) \sigma_{N N}^{\text {tot }} \delta(\boldsymbol{b})$, but note that it does not reproduce the $N N$ total elastic cross section. The $\sigma_{\mathrm{cc}}$ with Eq. (9) is called $\sigma_{\mathrm{cc}}^{\text {free }}$ in Ref. [12], where the zero-range approximation is employed.

When the target density is well known, $P_{\mathrm{cc}}^{\operatorname{dir}}(\boldsymbol{b})$ depends on only the projectile's proton density. To test the validity of Eqs. (8) and (9), we choose a ${ }^{12} \mathrm{C}$ target whose density
TABLE I. Charge-changing cross sections, given in units of mb, of ${ }^{12,13,14} \mathrm{C}$ on a ${ }^{12} \mathrm{C}$ target at $E \mathrm{MeV}$. The projectile's proton (rootmean-square) radius $r_{p}$ is obtained from the charge radius of Ref. [21] by taking into account the finite size effect of the nucleon as well as the so-called Darwin-Foldy term. See, e.g., Ref. [22].

\begin{tabular}{lcccc}
\hline \hline Projectile & $r_{p}(\mathrm{fm})$ & $\sigma_{\mathrm{cc}}^{\mathrm{dir}}$ & $\sigma_{\mathrm{cc}}$ (expt.) & $E / A$ \\
\hline${ }^{12} \mathrm{C}$ & 2.326 & 735 & $734 \pm 6[8]$ & 943 \\
& & 735 & $733 \pm 7[13]$ & 937 \\
${ }^{13} \mathrm{C}$ & 2.321 & 732 & $726 \pm 7[13]$ & 828 \\
${ }^{14} \mathrm{C}$ & 2.369 & 743 & $731 \pm 7[13]$ & 900 \\
\hline \hline
\end{tabular}

distribution is well known and take up the projectiles of ${ }^{12,13,14} \mathrm{C}$ whose proton radii $\left(r_{p}\right)$ are known. Assuming the harmonic-oscillator (HO) density distributions [19,20], we set the oscillator parameters to reproduce $r_{p}$. As compared in Table I, the $\sigma_{\text {cc }}^{\text {dir }}$ values agree with the measured cross sections quite well, which confirms the validity of our formulation.

With the increasing number of neutrons, it is likely that the proton orbits of the projectile isotopes are deeply bound and few states with $a \neq 0$ may not lead to the CCR. If that is the case, the $\sigma_{\mathrm{cc}}^{\mathrm{dir}}$ value calculated using Eq. (9) tends to be larger than experiment.

Figure 1 displays $\sigma_{\mathrm{cc}}^{\text {dir }}$ as well as $\sigma_{R}$ of ${ }^{12} \mathrm{C}+{ }^{12} \mathrm{C}$ as a function of $E$. Here $\sigma_{R}$ is calculated in the nucleon-target formalism in the Glauber model (NTG) [23,24], which performs slightly better than OLA. Both the magnitude and energy dependence of $\sigma_{R}$ or the interaction cross section $\left(\sigma_{I}\right)$ are reproduced very well. As for $\sigma_{\mathrm{cc}}^{\mathrm{dir}}$, the calculation reasonably well reproduces the measured cross sections [25]. The $\sigma_{\mathrm{cc}}$ data at $300 A-400 A \mathrm{MeV}$ are, however, very much scattered. More accurate data are needed to examine the energy dependence of $\sigma_{\text {cc }}$ before introducing the $E$-dependent factor $[4,5]$.

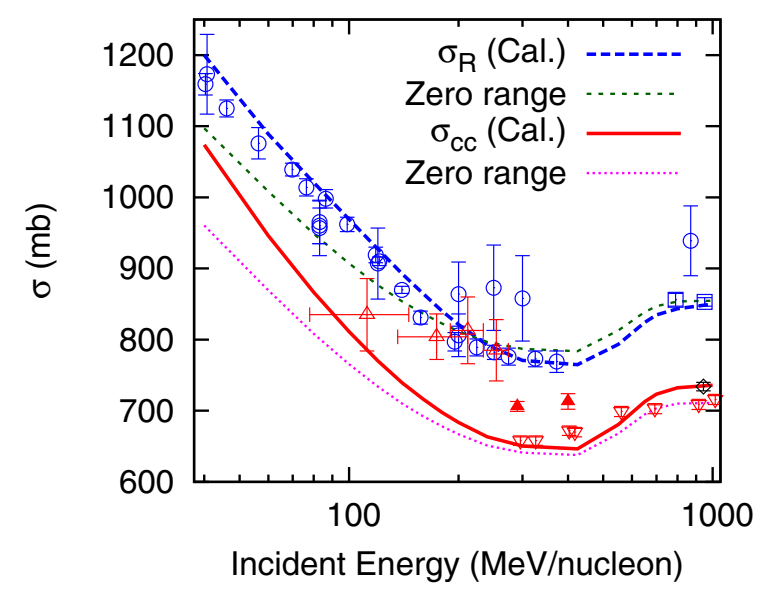

FIG. 1. Total reaction (or interaction) and charge-changing cross sections of ${ }^{12} \mathrm{C}$ on a ${ }^{12} \mathrm{C}$ target as a function of incident energy. Calculations are performed with the $\mathrm{HO}$ densities that give $r_{p}=$ $r_{n}=2.326 \mathrm{fm}$. Results with the zero-range profile functions are also drawn for comparison. References for the experimental data on $\sigma_{R}$ (open circle) and $\sigma_{I}$ (open rectangle) are quoted in Ref. [24]. The $\sigma_{\mathrm{cc}}$ data are taken from Ref. [8] for diamond, Ref. [25] for inverted triangle, Ref. [26] for closed triangle, and Ref. [27] for open triangle. 
As Table I shows, the ${ }^{14} \mathrm{C}+{ }^{12} \mathrm{C} \sigma_{\mathrm{cc}}$ is well reproduced at $900 A \mathrm{MeV}$. Therefore, the $\sigma_{\mathrm{cc}}^{\mathrm{dir}}$ value around $300 \mathrm{~A} \mathrm{MeV}$ should be smaller than $743 \mathrm{mb}$, as expected from the energy dependence of $\sigma_{p n}^{\text {tot }}$ and $\sigma_{p p}^{\text {tot }}$. Our prediction is $661 \mathrm{mb}$, whereas the experimental value at $287 \mathrm{~A} \mathrm{MeV}$ is $731 \pm 5 \mathrm{mb}$ [5], much larger than that expected theoretically.

Next we examine the $\sigma_{\mathrm{cc}}$ by a proton target. The direct process in this case turns out to underestimate the observed $\sigma_{\mathrm{cc}}$. We expect the projectile's neutrons to contribute to the cross sections. A most probable possibility is the following: the flux of the target proton attenuates due to the interaction with the projectile nucleus, a part of the lost proton flux is converted to the neutron flux, and that neutron leads to the CCR. A microscopic description of this ' $p-n$ exchange' mechanism is beyond the scope of this paper because we do not explicitly include neutron channels to describe the CCR. We attempt formulating this process called "indirect" within the eikonal approximation. To make the argument conventional, we use the inverse kinematics in which the (target) proton is impinging on the (projectile) nucleus.

The proton wave function modified from the plane wave is given by

$$
\Psi(\boldsymbol{r})=\exp \left(i k z+\frac{1}{i \hbar v} \int_{-\infty}^{z} d z^{\prime}\left[V_{p}^{(p)}\left(\boldsymbol{b}, z^{\prime}\right)+V_{p}^{(n)}\left(\boldsymbol{b}, z^{\prime}\right)\right]\right),
$$

where $\boldsymbol{r}=(\boldsymbol{b}, z), v$ is the proton velocity, and $V_{p}^{(N)}$ is the proton optical potential due to the protons $(N=p)$ or the neutrons $(N=n)$ in the nucleus. Note that the eikonal approximation enables us to obtain $V_{p}^{(N)}$ from $\left\langle 0_{P}\left|e^{i \chi_{p}^{(N)}}\right| 0_{P}\right\rangle$ with $\chi_{p}^{(N)}=$ $\sum_{i \in N} \chi_{N p}\left(b+s_{i}\right)[9,14,15]$. The $\sigma_{\mathrm{cc}}^{\text {dir }}$ for the proton-nucleus case is rewritten to a form of the so-called absorption cross section [15]

$$
\sigma_{\mathrm{cc}}^{\mathrm{dir}}=\int d \boldsymbol{b} \int_{-\infty}^{\infty} d z A_{p}^{(p)}(\boldsymbol{b}, z) D_{p}^{(p)}(\boldsymbol{b},-\infty, z),
$$

where

$$
A_{p / n}^{(N)}(\boldsymbol{b}, z)=-\frac{2}{\hbar v} \operatorname{Im} V_{p / n}^{(N)}(\boldsymbol{b}, z),
$$

and $D$ stands for the attenuation of the flux

$$
D_{p / n}^{(N)}\left(\boldsymbol{b}, z_{1}, z_{2}\right)=\exp \left(-\int_{z_{1}}^{z_{2}} d z^{\prime} A_{p / n}^{(N)}\left(\boldsymbol{b}, z^{\prime}\right)\right) .
$$

In the first step of the indirect process, the proton impinging from $z=-\infty$ reaches $z=z_{1}$, where the exchange occurs. In the second step, the neutron proceeds to $z=z_{2}$, keeping both the trajectory and velocity of the incident proton and is absorbed by the proton optical potential. Let $P_{n}\left(\boldsymbol{b}, z_{1}\right)$ denote the probability of the $p-n$ exchange per unit length. This exchange is considered the loss or disappearance of the proton due to the optical potential:

$$
P_{n}\left(\boldsymbol{b}, z_{1}\right)=P_{\mathrm{abs}}\left(\boldsymbol{b}, z_{1}\right) \frac{\rho_{P}^{(n)}\left(\boldsymbol{b}, z_{1}\right)}{\rho_{P}^{(p)}\left(\boldsymbol{b}, z_{1}\right)+\rho_{P}^{(n)}\left(\boldsymbol{b}, z_{1}\right)},
$$

where $P_{\mathrm{abs}}\left(\boldsymbol{b}, z_{1}\right)$ is the rate of the disappearance of the proton at $\left(\boldsymbol{b}, z_{1}\right)$ and the ratio of the densities stands for the probability of finding the neutron. With the use of Eq. (11), $P_{\mathrm{abs}}\left(\boldsymbol{b}, z_{1}\right)$ is obtained as $P_{\text {abs }}\left(\boldsymbol{b}, z_{1}\right) \delta z_{1} \approx\left|\Psi\left(\boldsymbol{b}, z_{1}\right)\right|^{2}-\left|\Psi\left(\boldsymbol{b}, z_{1}+\delta z_{1}\right)\right|^{2}$, leading to

$$
\begin{aligned}
P_{\mathrm{abs}}\left(\boldsymbol{b}, z_{1}\right)= & {\left[A_{p}^{(n)}\left(\boldsymbol{b}, z_{1}\right)+A_{p}^{(p)}\left(\boldsymbol{b}, z_{1}\right)\right] } \\
& \times D_{p}^{(n)}\left(\boldsymbol{b},-\infty, z_{1}\right) D_{p}^{(p)}\left(\boldsymbol{b},-\infty, z_{1}\right),
\end{aligned}
$$

While the neutron proceeds to $z_{2}$, the neutron probability is reduced by the absorption of $V_{n}^{(N)}$, resulting in

$$
\rho_{n}\left(\boldsymbol{b}, z_{1}, z_{2}\right)=P_{n}\left(\boldsymbol{b}, z_{1}\right) D_{n}^{(n)}\left(\boldsymbol{b}, z_{1}, z_{2}\right) D_{n}^{(p)}\left(\boldsymbol{b}, z_{1}, z_{2}\right) .
$$

The $\sigma_{\mathrm{cc}}^{\text {indir }}$ is obtained, similar to Eq. (12), as

$$
\sigma_{\mathrm{cc}}^{\text {indir }}=\int d \boldsymbol{b} P_{\mathrm{cc}}^{\mathrm{indir}}(\boldsymbol{b})
$$

with

$$
P_{\mathrm{cc}}^{\mathrm{indir}}(\boldsymbol{b})=\int_{-\infty}^{\infty} d z_{1} \int_{z_{1}}^{\infty} d z_{2} \rho_{n}\left(\boldsymbol{b}, z_{1}, z_{2}\right) A_{n}^{(p)}\left(\boldsymbol{b}, z_{2}\right) .
$$

The range $b$ in which $P_{\mathrm{cc}}^{\mathrm{indir}}(\boldsymbol{b})$ is appreciable is confined by that of $P_{\mathrm{cc}}^{\mathrm{dir}}(\boldsymbol{b})$ because of the trajectory assumption of the indirect process.

The $\sigma_{\mathrm{cc}}$ values of carbon isotopes $(A=12-19)$ for a proton target have been measured at the GSI fragment separator (FRS) facility with secondary beams of about $900 \mathrm{~A} \mathrm{MeV}$. The method of the experiment is the same as the previously published one $[7,8,13]$. A polyethylene target of $4.0 \mathrm{~g} / \mathrm{cm}^{2}$ in thickness was used and the $\sigma_{\mathrm{cc}}$ of a ${ }^{12} \mathrm{C}$ target were subtracted. The cross sections of the ${ }^{12} \mathrm{C}$ target were measured at the same time with the same experimental system using a graphite target of $4.0 \mathrm{~g} / \mathrm{cm}^{2}$ in thickness [13]. The obtained $\sigma_{\mathrm{cc}}$ are listed in Table II.

We display in Fig. $2 \sigma_{\mathrm{cc}}$ for $p+{ }^{12-19} \mathrm{C}$. The proton and neutron densities of carbon isotopes are given by the $\mathrm{HO}$ distributions, and their oscillator parameters are determined to reproduce both $\sigma_{\mathrm{cc}}$ [13] and $\sigma_{I}$ [28] data on the ${ }^{12} \mathrm{C}$ target. Here $\sigma_{I}$ is approximated by $\sigma_{R}$ calculated in the NTG approximation. The obtained proton, neutron, and matter radii are listed in Table III. The $r_{p}$ values of ${ }^{12,13,14} \mathrm{C}$ are in reasonable agreement with the empirical values of Table I. The difference is less than $3 \%$ for ${ }^{14} \mathrm{C}$. Though the $r_{p}$ values of the heavier isotopes do not change much as the neutron number increases, the $r_{n}$ values increase by about $0.3 \mathrm{fm}$ at ${ }^{15,16,19} \mathrm{C}$. This may indicate the effect of the $1 s$ neutron orbit. The isotope dependence of $r_{n}$ shows a pattern considerably different from

TABLE II. Charge-changing cross sections, given in units of mb, of carbon isotopes on a proton target at $E \mathrm{MeV}$.

\begin{tabular}{lccccccc}
\hline \hline Projectile & ${ }^{12} \mathrm{C}$ & ${ }^{13} \mathrm{C}$ & ${ }^{14} \mathrm{C}$ & ${ }^{15} \mathrm{C}$ & ${ }^{16} \mathrm{C}$ & ${ }^{17} \mathrm{C}$ & ${ }^{18} \mathrm{C}$ \\
\hline$\sigma_{\text {cc }}($ expt. $)$ & $214 \pm 7$ & $227 \pm 7$ & $222 \pm 9$ & $224 \pm 5$ & $224 \pm 4$ & $222 \pm 11$ & $228 \pm 5$ \\
$E / A$ & 926 & 815 & 889 & 896 & 897 & 970 & 886 \\
\hline \hline
\end{tabular}




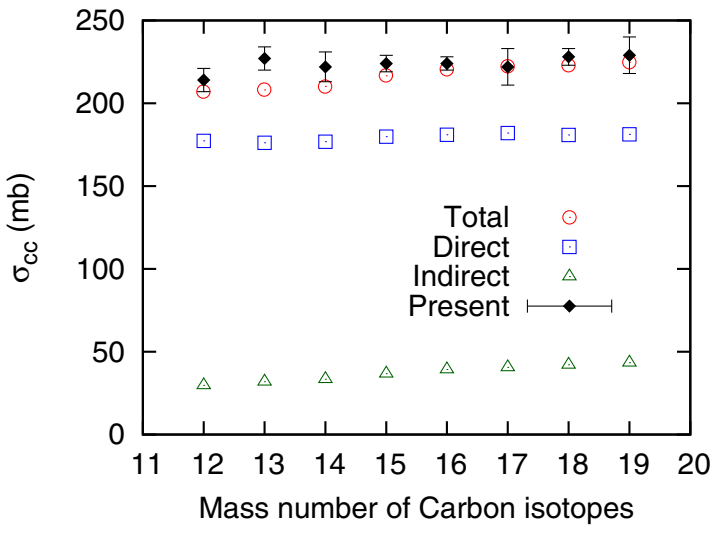

FIG. 2. Charge-changing cross sections of carbon isotopes on a proton target at about $900 \mathrm{~A} \mathrm{MeV}$.

that of Refs. [24,29]. As seen in Fig. 2, our theory satisfactorily reproduces the measured $\sigma_{\mathrm{cc}}$. The magnitude of $\sigma_{\mathrm{cc}}^{\text {indir }}$ increases with the neutron number, ranging from 30 to $50 \mathrm{mb}$, which is on the order of the cross section missed by the direct process. The $r_{p}$ and $r_{n}$ values of Table III, albeit extracted based on the $\mathrm{HO}$ density distributions, are considered reasonable in that they simultaneously reproduce all the $\sigma_{I}$ and $\sigma_{\mathrm{cc}}$ data on both ${ }^{12} \mathrm{C}$ and proton targets. Higher-order processes such as $p \rightarrow n \rightarrow p$ are expected to make a negligible contribution at high incident energy.

The energy dependence of $\sigma_{\mathrm{cc}}$ for a proton target is of considerable importance to understanding the interstellar production of secondary fragments during cosmic-ray propagation [30]. Figure 3(a) exhibits the $p+{ }^{12} \mathrm{C} \sigma_{\mathrm{cc}}$ as a function of energy. The energy dependence of $\sigma_{\mathrm{cc}}^{\text {dir }}$ on a proton target is basically determined by that of $\sigma_{p p}^{\text {tot }}$. The indirect process is, however, influenced by $\sigma_{p n}^{\text {tot }}$ as well and its energy dependence becomes important. For example, $\sigma_{p n}^{\text {tot }}$ is slightly smaller than $\sigma_{p p}^{\text {tot }}$ at $E>600 \mathrm{MeV}$ but becomes larger than $\sigma_{p p}^{\text {tot }}$ below that energy, e.g., 2.5 times larger at $E=100 \mathrm{MeV}$. The sum of $\sigma_{\mathrm{cc}}^{\text {dir }}$ and $\sigma_{\mathrm{cc}}^{\text {indir }}$ reasonably well reproduces the energy dependence of the $\sigma_{\mathrm{cc}}$, especially the present high-energy data and the low-energy data of Ref. [26].

The magnitude of $\sigma_{\mathrm{cc}}^{\mathrm{dir}}$ on a proton target reflects the proton distribution of the projectile nucleus. However, the magnitude of $\sigma_{\mathrm{cc}}^{\text {indir }}$ depends on the neutron distribution as well. The ratio of both contributions alters with increasing neutron numbers of isotopes. Figure 3(b) exhibits the energy dependence of both contributions to $p+{ }^{19} \mathrm{C} \sigma_{\mathrm{cc}}$. A comparison between $p+{ }^{12} \mathrm{C}$ and $p+{ }^{19} \mathrm{C}$ cases indicates that the $\sigma_{\mathrm{cc}}^{\mathrm{dir}}$ values change little

TABLE III. Proton, neutron, and matter radii, $r_{p}, r_{n}$, and $r_{m}$, of carbon isotopes determined from $\sigma_{\mathrm{cc}}$ [13] and $\sigma_{I}$ [28] data on a ${ }^{12} \mathrm{C}$ target.

\begin{tabular}{lcccccccc}
\hline \hline Isotope & ${ }^{12} \mathrm{C}$ & ${ }^{13} \mathrm{C}$ & ${ }^{14} \mathrm{C}$ & ${ }^{15} \mathrm{C}$ & ${ }^{16} \mathrm{C}$ & ${ }^{17} \mathrm{C}$ & ${ }^{18} \mathrm{C}$ & ${ }^{19} \mathrm{C}$ \\
\hline$r_{p}(\mathrm{fm})$ & 2.32 & 2.30 & 2.31 & 2.37 & 2.40 & 2.42 & 2.39 & 2.40 \\
$r_{n}(\mathrm{fm})$ & 2.32 & 2.34 & 2.34 & 2.64 & 2.93 & 2.93 & 3.07 & 3.46 \\
$r_{m}(\mathrm{fm})$ & 2.32 & 2.32 & 2.33 & 2.54 & 2.74 & 2.76 & 2.86 & 3.16 \\
\hline \hline
\end{tabular}

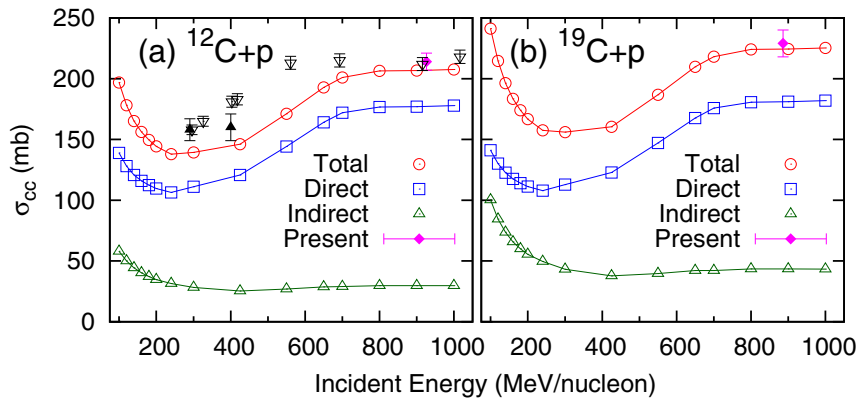

FIG. 3. Energy dependence of charge-changing cross sections of (a) ${ }^{12} \mathrm{C}$ and (b) ${ }^{19} \mathrm{C}$ on a proton target. The data are taken from Ref. [25] for open inverted triangles and from Ref. [26] for closed triangles.

but the indirect process gives considerably larger contribution in the $p+{ }^{19} \mathrm{C}$ CCR. A measurement of $\sigma_{\mathrm{cc}}$ as a function of energy will be important for both assessing the validity of our CCR model and extracting information on the neutron density of ${ }^{19} \mathrm{C}$.

Figure 4 compares the reaction probabilities of ${ }^{12,19} \mathrm{C}$ at $1000 A$ and $100 A \mathrm{MeV}$. Because the $r_{p}$ values of both nuclei are almost the same, their direct probabilities change only little. The difference in the magnitude of $P_{\mathrm{cc}}^{\mathrm{dir}}$ at $1000 \mathrm{~A}$ and $100 \mathrm{~A} \mathrm{MeV}$ is due to the energy dependence of $\sigma_{p p}^{\text {tot }}$. On the other hand, $P_{\mathrm{cc}}^{\text {indir }}$ shows interesting behavior. Since the $r_{n}$ value of ${ }^{19} \mathrm{C}$ is by about $1 \mathrm{fm}$ larger than that of ${ }^{12} \mathrm{C}$, its probability $P_{\mathrm{cc}}^{\text {indir }}$ extends to larger $b$ values. The magnitude of $P_{\mathrm{cc}}^{\text {indir }}$ is controlled by the $p-n$ interaction. The $\sigma_{p n}^{\text {tot }}$ value at $100 \mathrm{MeV}$ is two times larger than that at $1000 \mathrm{MeV}$, which makes the indirect process contribute appreciably to the $\sigma_{\mathrm{cc}}$. As already noted, however, $P_{\mathrm{cc}}^{\text {indir }}$ of ${ }^{19} \mathrm{C}$ even at $100 \mathrm{~A} \mathrm{MeV}$ does not exceed the range of its $P_{\mathrm{cc}}^{\mathrm{dir}}$.

To conclude, we have developed a method of calculating charge-changing cross sections in order to probe charge distributions. We have compared our calculations with recent new high-energy data measured at the FRS facility of GSI. With the input of the known proton radii for ${ }^{12,13,14} \mathrm{C}$, we have confirmed that the direct process based on the Glauber theory fairly well reproduces the cross sections on a ${ }^{12} \mathrm{C}$

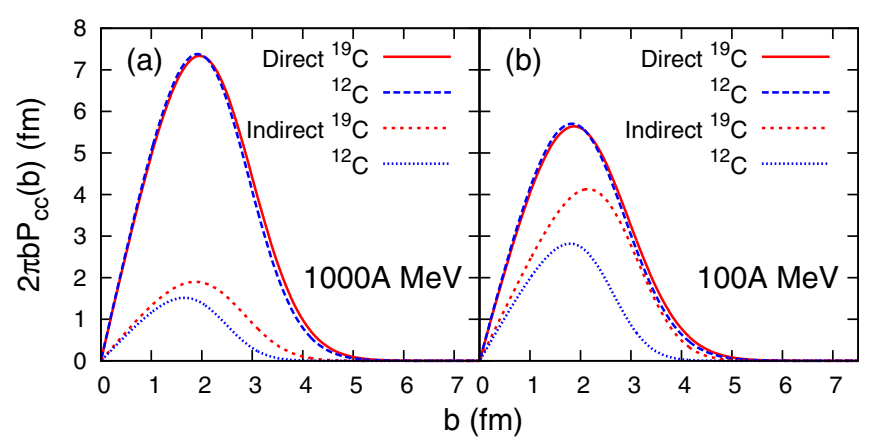

FIG. 4. Impact-parameter dependence of the direct and indirect charge-changing probabilities for ${ }^{12} \mathrm{C}$ and ${ }^{19} \mathrm{C}$ on a proton target at (a) $1000 \mathrm{~A}$ and (b) $100 \mathrm{~A} \mathrm{MeV}$. 
target. We find that the charge-changing cross sections of ${ }^{12-19} \mathrm{C}$ on a proton target are all underestimated by the direct process alone. The indirect process in which the neutrons participate in the charge-changing reaction is formulated in the eikonal approximation. This parameter-free model has succeeded in reproducing the new data. As a future study, it will be interesting to apply the theory to charge-changing cross sections for other projectiles with different incident energies.

The work was in part supported by JSPS KAKENHI Grants No. 23224008, No. 24540261, No. 25800121, and No. $15 \mathrm{~K} 05072$.
[1] T. Suda and M. Wakasugi, Prog. Part. Nucl. Phys. 55, 417 (2005).

[2] A. N. Antonov et al., Nucl. Instr. and Meth. A 637, 60 (2011).

[3] I. Tanihata, H. Savajols, and R. Kanungo, Prog. Part. Nucl. Phys. 68, 215 (2013).

[4] T. Yamaguchi, M. Fukuda, S. Fukuda, G. W. Fan, I. Hachiuma, M. Kanazawa, A. Kitagawa, T. Kuboki, M. Lantz, M. Mihara, M. Nagashima, K. Namihira, D. Nishimura, Y. Okuma, T. Ohtsubo, S. Sato, T. Suzuki, M. Takechi, and W. Xu, Phys. Rev. C 82, 014609 (2010).

[5] T. Yamaguchi, I. Hachiuma, A. Kitagawa, K. Namihira, S. Sato, T. Suzuki, I. Tanihata, and M. Fukuda, Phys. Rev. Lett. 107, 032502 (2011).

[6] A. Ozawa et al., Phys. Rev. C 89, 044602 (2014).

[7] A. Estradé et al., Phys. Rev. Lett. 113, 132501 (2014).

[8] S. Terashima, I. Tanihata, R. Kanungo, A. Estradé, W. Horiuchi, F. Ameil, J. Atkinson, Y. Ayyad, D. Cortina-Gil, I. Dillmann, A. Evdokimov, F. Farinon, H. Geissel, G. Guastalla, R. Janik, M. Kimura, R. Knoebel, J. Kurcewicz, Yu. A. Litvinov, M. Marta, M. Mostazo, I. Mukha, T. Neff, C. Nociforo, H. J. Ong, S. Pietri, A. Prochazka, C. Scheidenberger, B. Sitar, Y. Suzuki, M. Takechi, J. Tanaka, J. Vargas, J. S. Winfield, and H. Weick, Prog. Theor. Exp. Phys. 2014, 101D02 (2014).

[9] R. J. Glauber, Lectures in Theoretical Physics, edited by W. E. Brittin and L. G. Dunham (Interscience, New York, 1959), Vol. 1, p. 315 .

[10] W. Horiuchi, Y. Suzuki, and T. Inakura, Phys. Rev. C 89, 011601(R) (2014).

[11] W. Horiuchi, S. Hatakeyama, S. Ebata, and Y. Suzuki, Phys. Rev. C 93, 044611 (2016).

[12] A. Bhagwat and Y. K. Gambhir, Phys. Rev. C 69, 014315 (2004).
[13] R. Kanungo et al. (unpublished).

[14] K. Yabana, Y. Ogawa, and Y. Suzuki, Nucl. Phys. A 539, 295 (1992)

[15] Y. Suzuki, R. G. Lovas, K. Yabana, and K. Varga, Structure and Reactions of Exotic Nuclei (Taylor \& Francis, London, 2003).

[16] P. J. Karol, Phys. Rev. C 11, 1203 (1975).

[17] L. Ray, Phys. Rev. C 20, 1857 (1979).

[18] B. Abu-Ibrahim, W. Horiuchi, A. Kohama, and Y. Suzuki, Phys. Rev. C 77, 034607 (2008).

[19] H. De Vries, C. W. De Jager, and C. De Vries, At. Data Nucl. Data Tables 36, 495 (1987).

[20] B. Abu-Ibrahim, S. Iwasaki, W. Horiuchi, A. Kohama, and Y. Suzuki, J. Phys. Soc. Jpn. 78, 044201 (2009).

[21] I. Angeli and K. P. Marinova, At. Data Nucl. Data Tables 99, 69 (2013).

[22] W. Nörtershäuser et al., Phys. Rev. Lett. 102, 062503 (2009).

[23] B. Abu-Ibrahim and Y. Suzuki, Phys. Rev. C 61, 051601(R) (2000).

[24] W. Horiuchi, Y. Suzuki, B. Abu-Ibrahim, and A. Kohama, Phys. Rev. C 75, 044607 (2007).

[25] W. R. Webber, J. C. Kish, and D. A. Schrier, Phys. Rev. C 41, 520 (1990).

[26] C. Zeitlin, C. Zeitlin, S. Guetersloh, L. Heilbronn, J. Miller, A. Fukumura, Y. Iwata, and T. Murakami, Phys. Rev. C 76, 014911 (2007).

[27] A. N. Golovchenko, J. Skvarč, N. Yasuda, M. Giacomelli, S. P. Tretyakova, R. Ilić, R. Bimbot, M. Toulemonde, and T. Murakami, Phys. Rev. C 66, 014609 (2002).

[28] A. Ozawa, T. Suzuki, and I. Tanihata, Nucl. Phys. A 693, 32 (2001)

[29] Y. Kanada-Eny'o, Phys. Rev. C 91, 014315 (2015).

[30] W. R. Webber, J. C. Kish, and D. A. Schrier, Phys. Rev. C 41, 533 (1990). 